\title{
SBF1 mutations associated with autosomal recessive axonal neuropathy with cranial nerve involvement
}

Andreea Manole, ${ }^{* 1,2}$ Alejandro Horga, ${ }^{* 1}$ Josep Gamez, ${ }^{3}$ Nuria Raguer, ${ }^{4}$ Maria Salvado, ${ }^{3}$ Beatriz San Millán, ${ }^{5}$ Carmen Navarro, ${ }^{5}$ Alan Pittmann, ${ }^{2}$ Mary M. Reilly, ${ }^{1}$ Henry Houlden. ${ }^{1,2}$

${ }^{1}$ MRC Centre for Neuromuscular Diseases, UCL Institute of Neurology, Queen Square, London, UK. ${ }^{2}$ Department of Molecular Neuroscience, UCL Institute of Neurology, Queen Square, London, United Kingdom. ${ }^{3}$ Neuromuscular Disorders Unit, Department of Neurology, Hospital Universitari Vall d'Hebron and Universitat Autònoma de Barcelona, VHIR, Barcelona, Spain. ${ }^{4}$ Department of Neurophysiology, Hospital Universitari Vall d'Hebron and Universitat Autònoma de Barcelona, VHIR, Barcelona, Spain. ${ }^{5}$ Department of Neuropathology. Complejo Hospitalario Universitario de Vigo, Vigo, Spain.

*These authors contributed equally to the study.

Words in manuscript: 1372

Words in abstract: 95

Number of tables: 1

Number of figures: 1

Number of references: 14

Supplementary material: PDF file

Correspondence to: Dr Josep Gamez. Neuromuscular Disorders Unit, Department of Neurology, Hospital Universitari Vall d'Hebron, Passeig Vall d'Hebron, 119-135, 08035 Barcelona, Spain. Email: josepgamez.bcn@gmail.com. Tel: + 34 932746000. Fax: +34 932110912. 


\begin{abstract}
Biallelic mutations in the SBF1 gene have been identified in one family with demyelinating Charcot-MarieTooth disease (CMT4B3) and two families with axonal neuropathy and additional neurological and skeletal features. Here we describe novel sequence variants in SBF1 (c.1168C $>\mathrm{G}$ and c.2209_2210del) as the potential causative mutations in two siblings with severe axonal neuropathy, hearing loss, facial weakness and bulbar features. Pathogenicity of these variants is supported by co-segregation and in silico analyses and evolutionary conservation. Our findings suggest that SBF1 mutations may cause a syndromic form of autosomal recessive axonal neuropathy (AR-CMT2) in addition to CMT4B3.
\end{abstract}

\title{
ABBREVIATIONS
}

CMT = Charcot-Marie-Tooth disease; MAF = minor allele frequency.

\section{KEYWORDS}

Charcot-Marie-Tooth disease; inherited neuropathy; MTMR2; SBF1; SBF2; whole-exome sequencing. 


\section{INTRODUCTION}

Charcot-Marie-Tooth disease (CMT), a genetically heterogeneous disorder of the peripheral nerves, is classically divided into demyelinating or axonal subtypes based on neurophysiology data. Mutations in genes encoding myotubularin-related proteins (MTMR2 and SBF2) are associated with autosomal recessive forms of demyelinating CMT (CMT4B1 and CMT4B2, respectively). ${ }^{1,2}$ Mutations in the SBF1 gene, which encodes another member of the myotubularin family, have been identified in one Korean family with autosomal recessive demyelinating CMT (CMT4B3) but also in one Saudi Arabian and one Syrian family with axonal neuropathy, multiple cranial neuropathies, intellectual disability and skeletal features including microcephaly. ${ }^{3-7}$ In this paper, we describe novel variants in $S B F 1$ as the potential causative mutations in two siblings with severe axonal neuropathy, hearing loss, facial weakness and bulbar features. Our findings support the previous observation that SBF1 mutations may cause a syndromic form of axonal neuropathy in addition to CMT4B3.

\section{METHODS}

Two brothers with peripheral neuropathy were investigated. Informed consent was obtained from all individuals and the institutional review boards at the participating medical centres approved the study. Individuals underwent clinical and instrumental assessments during the routine diagnostic process. Neurophysiological studies, MRI scans and skin, muscle and nerve biopsies were performed using standard methods. Genomic DNA from the two affected individuals and four unaffected relatives was used for molecular genetic analyses. For detailed methods see the online supplementary material.

\section{RESULTS}

Clinical features. The proband and his affected brother (II:3 and II:2; Fig. 1A) were the third and second children of healthy, non-consanguineous parents of Spanish descent. Both walked independently at age 14 months. At ages 4 and 9 years, respectively, they were noted to have an unsteady gait and subsequently developed slowly progressive, distal-predominant, muscle weakness and sensory loss in their limbs. They lost ambulation in their mid- to late-30s. In their late 40s, on neurological examination, they had gaze-evoked nystagmus (plus delayed initiation of horizontal saccades and mild ophthalmoparesis in patient II:3), bilateral hearing loss, upper and lower facial weakness, bulbar features including tongue weakness, dysarthria, dysphagia and reduced or absent gag reflex, lumbar hyperlordosis, mild pes cavus, distal and proximal muscle weakness and atrophy and marked sensory impairment in their limbs (Table 1 and Fig. 1C-D). They were unable to stand unaided. Their IQ was 85 and 83, respectively.

Nerve conduction studies were consistent with a severe length-dependent, motor and sensory axonal neuropathy with median and ulnar motor nerve conduction velocities ranging between 49 and $61 \mathrm{~m} / \mathrm{s}$ (supplementary Table 1). Compound muscle action potentials of the facial nerves were markedly reduced in patient II:3. Needle EMG showed neurogenic motor unit action potentials in the limbs and facial muscles (supplementary Table 2). In patient II:3, brainstem auditory evoked potentials were consistent with left sensorineural cochlear impairment and visual evoked potentials showed no abnormalities.

Creatine kinase levels were $290 \mathrm{IU} / \mathrm{L}$ in patient II:3 and $420 \mathrm{IU} / \mathrm{L}$ in patient II:2 (normal values $\leq 195 \mathrm{IU} / \mathrm{L}$ ). Brain MRIs in both cases demonstrated mild cerebellar atrophy (Fig. 1E-F). Skin, tibialis anterior muscle and sural nerve biopsies were performed in patient II:3 at age 34 years. Skin biopsy was unremarkable. Muscle biopsy showed features of longstanding neurogenic atrophy. Nerve biopsy revealed diffuse loss of myelinated axons of all diameters, few regenerating clusters and occasional moderately-thin myelin sheaths; no onion bulbs were observed. The final histopathological diagnosis was axonal neuropathy (Fig. 1G-H). Muscle biopsy of patient II:2 at age 32 years also showed features of longstanding neurogenic atrophy.

Genetic results. Genetic analysis of the 17p11.2 chromosome region and direct sequencing of GJB1, AAAS, SLC52A2 and SLC52A3 genes revealed no pathogenic variants. Genetic tests for spinal muscular atrophy, Kennedy's disease, Friedreich ataxia, spinocerebellar ataxia types 1, 2, 3, 6, 7, 8, 12 and 17, dentatorubralpallidoluysian atrophy, and fragile X-associated tremor/ataxia syndrome were also negative. To identify the underlying genetic cause, we applied whole-exome sequencing on the proband (II:3) and one unaffected brother (II:1). Analysis focused on nonsynonymous, splice-site and coding indel variants with a minor allele frequency (MAF) of $<0.5 \%$ in the Exome Aggregation Consortium (ExAC; exac.broadinstitute.org), Exome Variant Server 
(EVS; evs.gs.washington.edu) and 1000 Genomes databases (1000G; www.1000genomes.org). From a total of 613 variants that met these filtering criteria in the proband, 186 variants in 60 genes co-segregated under an autosomal recessive model and 2 variants in 2 genes under a X-linked model. Of these 188 variants, only 3 involved a gene associated with inherited neuropathy, i.e. the SBF1 gene (supplementary Table 3). No potentially pathogenic sequence variants were detected in known genes associated with cerebellar ataxia or hearing loss.

The SBF1 variants were validated by Sanger sequencing (supplementary Fig.). Segregation analysis confirmed that both affected siblings and their unaffected father were heterozygotes for the SBF1 variants c.2209_2210del and c.5197C $>\mathrm{T}$, indicating that these two variants were located in the same allele, and that all affected and unaffected siblings and their mother were heterozygous for the SBF1 variant c.1168C $>\mathrm{G}$ (Fig. 1A).

c.2209_2210del and c.1168C >G are novel variants not previously reported in public databases (supplementary Table 4). c.1168C >G, located in exon 11 of the SBF1 gene (41 exons; Ensembl transcript ENST00000380817), leads to the substitution of positively charged arginine for neutral glycine at codon 390 (p.Arg390Gly), affects a highly conserved nucleotide (Fig. 1B) and amino acid and is predicted as being deleterious by pathogenicity prediction tools. c.2209_2210del, located in exon 19, creates a frame shift starting at codon 737 that ends in a premature stop codon 2 positions downstream (p.Leu737Glufs*3).

c.5197C $>\mathrm{T}$ is reported in the dbSNP database (rs199972466) and is present in 62 individuals, including one homozygote, in the ExAC database (MAF $=0.053 \%$; supplementary Table 4). This variant is located in exon 38 and leads to the substitution of positively charged arginine for neutral cysteine at codon 1733 (p.Arg1733Cys). c.5197C $>\mathrm{T}$ is predicted as being deleterious; however, in the two affected individuals from the present study, this variant was in cis with c.2209_2210del, which causes a premature stop codon 994 amino acids upstream. Thus, the contribution of c.5197C $>$ T to the observed phenotype is uncertain.

\section{DISCUSSION}

Using whole-exome sequencing in two siblings with a severe motor and sensory axonal neuropathy, hearing loss, facial weakness and bulbar features we have identified two novel compound heterozygous variants in SBF1 as the probable causative mutations.

Myotubularins comprise a group of catalytically active and inactive proteins involved in membrane trafficking and endocytosis. ${ }^{8,9}$ Inactive myotubularins such as SBF1 and SBF2 interact with and regulate their active homologues by heterodimerization, and coiled-coil domains seem to be crucial for this interaction. ${ }^{8-10}$ SBF1 interacts with the MTMR2 lipid phosphatase and deletion of the coiled-coil domain of SBF1 leads to an altered cellular localization of MTMR2 in vitro. ${ }^{10}$ The frameshift mutation p.Leu737Glufs*3 detected in our family may result in a truncated SBF1 protein lacking the coiled-coil domain, which could affect the activity and distribution of MTMR2. Alternatively, the mutation may cause lack of expression due to nonsense-mediated decay. Of note, nonsense and frameshift mutations are common in SBF2-related CMT4B2., ${ }^{2,11,12}$

Both SBF1 and SBF2 but not MTMR2 contain DENN domains involved in regulation of Rab GTPases, which are in turn central regulators of membrane trafficking..$^{13}$ Mutations affecting the dDENN motif of the DENN domain or nearby amino acid residues have been identified in two families with CMT4B (p.Met417Val in SBF1 and p.Leu351_Glu432del in SBF2) and syndromic forms of axonal neuropathy (p.Leu335Pro and p.Asp443Asn in SBF1). ${ }^{3-7,14}$ The novel variant p.Arg390Gly detected in our family is also located within the dDENN motif of SBF1, which suggests functional relevance.

Our cases have several similarities with the previously reported Saudi Arabian and Syrian families carrying the homozygous SBF1 variants p.Asp443Asn and p.Leu335Pro, respectively (clinical features summarized in supplementary Table 5). ${ }^{4-7}$ Ophthalmoparesis, facial weakness, dysarthria and dysphagia were present in all families with variable frequency and severity, and neurophysiological exam was consistent with an axonal neuropathy in all individuals although sensory and motor nerve conduction velocities were mildly decreased in one case. Some of the features observed in the previously reported families, however, including strabismus, microcephaly and moderate-to-severe intellectual disability, were absent in our cases. We believe that these three families share a new syndromic form of autosomal recessive axonal neuropathy (AR-CMT2) with multiple cranial neuropathies. Nevertheless, given the phenotypic variability and the previous association of SBF1 
mutations with CMT4B3, additional studies are needed to further define the clinical spectrum of SBF1-related neuropathies.

\section{COMPLIANCE WITH ETHICAL STANDARDS}

Ethical approval All procedures performed in studies involving human participants were in accordance with the ethical standards of the institutional and/or national research committee and with the 1964 Helsinki declaration and its later amendments or comparable ethical standards.

Informed consent Informed consent was obtained from all individual participants included in the study. Additional informed consent was obtained from all individual participants for whom identifying information is included in this article.

Conflict of interest The authors declare that they have no conflict of interest.

\section{REFERENCES}

1. Bolino A, Muglia M, Conforti FL, et al. Charcot-Marie-Tooth type 4B is caused by mutations in the gene encoding myotubularin-related protein-2 (2000). Nat Genet 25:17-19.

2. Azzedine H, Bolino A, Taieb T, et al. Mutations in MTMR13, a new pseudophosphatase homologue of MTMR2 and Sbf1, in two families with an autosomal recessive demyelinating form of Charcot-MarieTooth disease associated with early-onset glaucoma (2003). Am J Hum Genet 72:1141-1153.

3. Nakhro K, Park JM, Hong YB, et al. SET binding factor 1 (SBF1) mutation causes Charcot-MarieTooth disease type 4B3 (2013). Neurology 81:165-173.

4. Bohlega S, Alazami AM, Cupler E, Al-Hindi H, Ibrahim E, Alkuraya FS. A novel syndromic form of sensory-motor polyneuropathy is linked to chromosome 22q13.31-q13.33 (2011). Clin Genet 79:193195.

5. Alazami AM, Alzahrani F, Bohlega S, Alkuraya FS. SET binding factor 1 (SBF1) mutation causes Charcot-Marie-tooth disease type 4B3 (2014). Neurology 82:1665-1666.

6. Mégabarné A, Dorison N, Rodriguez D, Tamraz J. Multiple cranial nerve neuropathies, microcephaly, neurological degeneration, and "fork and bracket sign" in the MRI: a distinct syndrome (2010). Am J Med Genet A 152A: 2297-2300.

7. Romani M, Mehawej C, Mazza T, Mégarbané A, Valente EM. "Fork and bracket" syndrome expands the spectrum of SBF1-related sensory motor polyneuropathies (2016). Neurol Genet 2:e61.

8. Laporte J, Bedez F, Bolino A, Mandel JL. Myotubularins, a large disease-associated family of cooperating catalytically active and inactive phosphoinositides phosphatases (2003). Hum Mol Genet 12:R285-292.

9. Hnia K, Vaccari I, Bolino A, Laporte J. Myotubularin phosphoinositide phosphatases: cellular functions and disease pathophysiology (2012). Trends Mol Med 18:317-327.

10. Kim SA, Vacratsis PO, Firestein R, Cleary ML, Dixon JE. Regulation of myotubularin-related (MTMR)2 phosphatidylinositol phosphatase by MTMR5, a catalytically inactive phosphatase (2003). Proc Natl Acad Sci USA 100:4492-4497.

11. Hirano R, Takashima H, Umehara F, et al. SET binding factor 2 (SBF2) mutation causes CMT4B with juvenile onset glaucoma (2004). Neurology 63:577-580.

12. Baets J, Deconinck T, De Vriendt E, et al. Genetic spectrum of hereditary neuropathies with onset in the first year of life (2011). Brain 134:2664-2676.

13. Marat AL, Dokainish H, McPherson PS. DENN domain proteins: regulators of Rab GTPases (2011). J Biol Chem 286:13791-13800. 
14. Senderek J, Bergmann C, Weber S, et al. Mutation of the SBF2 gene, encoding a novel member of the myotubularin family, in Charcot-Marie-Tooth neuropathy type 4B2/11p15 (2003). Hum Mol Genet 12:349-356. 
Table 1. Clinical features of patients II:3 and II:2.

\begin{tabular}{|c|c|c|}
\hline Patient & II:3 & II:2 \\
\hline Gender & Male & Male \\
\hline Age of onset & $4 \mathrm{y}$ & $9 \mathrm{y}$ \\
\hline Age when wheelchair bound & $38 \mathrm{y}$ & $36 \mathrm{y}$ \\
\hline Age at examination & $48 \mathrm{y}$ & $49 \mathrm{y}$ \\
\hline First symptoms & Gait difficulties & Gait difficulties \\
\hline \multicolumn{3}{|l|}{ Cranial nerves } \\
\hline Hearing loss & Bilateral & Bilateral \\
\hline Facial weakness & Bilateral, asymmetric & Bilateral, asymmetric \\
\hline Ocular movements & $\begin{array}{l}\text { Horizontal-gaze evoked } \\
\text { nystagmus, delayed initiation } \\
\text { of horizontal saccades and } \\
\text { ophthalmoparesis }\end{array}$ & $\begin{array}{l}\text { Mild horizontal gaze-evoked } \\
\text { nystagmus }\end{array}$ \\
\hline Tongue involvement & Weakness & Weakness, atrophy \\
\hline Dysphagia & Yes & Yes \\
\hline Dysarthria & Yes & Yes \\
\hline Gag reflex & Absent & Diminished \\
\hline Neck flexion & Mild weakness & Mild weakness \\
\hline \multicolumn{3}{|l|}{ Limb involvement } \\
\hline Muscle tone & Reduced & Reduced \\
\hline Muscle atrophy & Moderate, distal>proximal & Moderate, distal>proximal \\
\hline \multicolumn{3}{|l|}{ Muscle weakness* } \\
\hline Upper limb & $\begin{array}{l}\text { Distal 3-4 } \\
\text { Proximal 3-4 } \\
\text { Shoulder abduction } 3 \\
\text { Pectoral muscles } 3\end{array}$ & $\begin{array}{l}\text { Distal } 2-4 \\
\text { Proximal } 3 \\
\text { Shoulder abduction } 1 \\
\text { Pectoral muscles } 3\end{array}$ \\
\hline Lower limb & $\begin{array}{l}\text { No movement except } \\
\text { for hip abduction } 3\end{array}$ & $\begin{array}{l}\text { No movement except } \\
\text { for knee extension 1-3 } \\
\text { and hip abduction } 3\end{array}$ \\
\hline \multicolumn{3}{|l|}{ Sensory loss } \\
\hline Pinprick & Reduced to wrists / ankles & Reduced to wrists / ankles \\
\hline Vibration & Absent to elbows / knees & Absent to elbows / knees \\
\hline Joint position & Absent at toes & Absent at toes \\
\hline Deep tendon reflexes & Absent & Absent \\
\hline Plantar responses & No response & No response \\
\hline Limb ataxia & Sensory ataxia & Sensory ataxia \\
\hline Gait pattern & $\mathrm{n} / \mathrm{e}$ & $\mathrm{n} / \mathrm{e}$ \\
\hline Romberg's test & $\mathrm{n} / \mathrm{e}$ & $\mathrm{n} / \mathrm{e}$ \\
\hline Intelligence quotient & 85 & 83 \\
\hline Respiratory involvement & No & Yes, NIV \\
\hline Other features & $\begin{array}{l}\text { Lumbar hyperlordosis } \\
\text { Mild pes cavus } \\
\text { Gynecomastia }\end{array}$ & $\begin{array}{l}\text { Lumbar hyperlordosis } \\
\text { Mild pes cavus } \\
\text { Gynecomastia }\end{array}$ \\
\hline
\end{tabular}


*Muscle strength according to the Medical Research Council (MRC) grading scale. n/e = not evaluable (unable to perform); NIV = non-invasive ventilation. 


\section{FIGURE LEGEND}

Figure 1. (A) Family pedigree and segregation of SBF1 variants; genotypes are indicated below tested individuals $(\mathrm{n} / \mathrm{t}=$ not tested). (B) Structural conservation of the relevant amino acid residues (Arg390 and Leu737) in SBF1 across 7 species (single letters = amino acid residues; black = identical; grey = conserved substitution); conservation among species of the affected amino acid residues was determined using Ensembl to retrieve the sequences and ClustalW2 software for multiple sequence alignment. (C, D) Clinical images of patients II:2 (C) and II:3 (D) showing asymmetric facial involvement at rest (left images) and incomplete eye closure (right images); note the preserved tongue muscle bulk in patient II:3 and tongue atrophy in patient II:2. (E, F) Axial brain MRI images of patient II:2 (T1-weighted image) and patient II:3 (T2-weighted image) showing atrophy of the cerebellar vermis. (G, H) Histopathological findings in sural nerve biopsy of patient II:3; transverse semi-thin section (G; Toulidine blue stain, magnification x400) and electron micrographs (H; original magnification x6200) showing decreased number of myelinated axons of all diameters and occasional moderately-thin myelin sheaths. 
A



c.[2209_2210del; 5197C>T]

c. $1168 \mathrm{C}>\mathrm{G}$

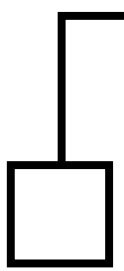

II:1

c. $1168 \mathrm{C}>\mathrm{G}$

B

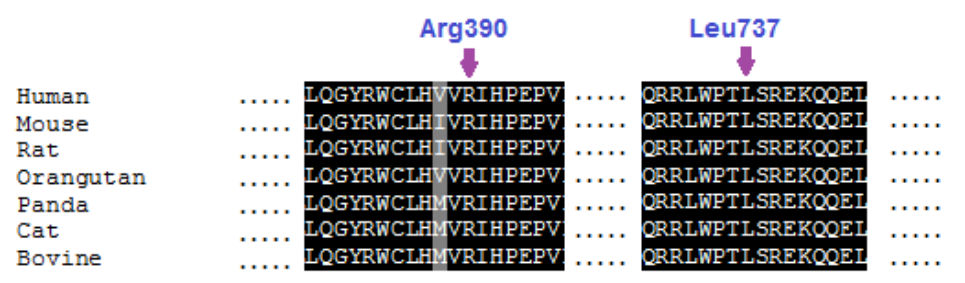
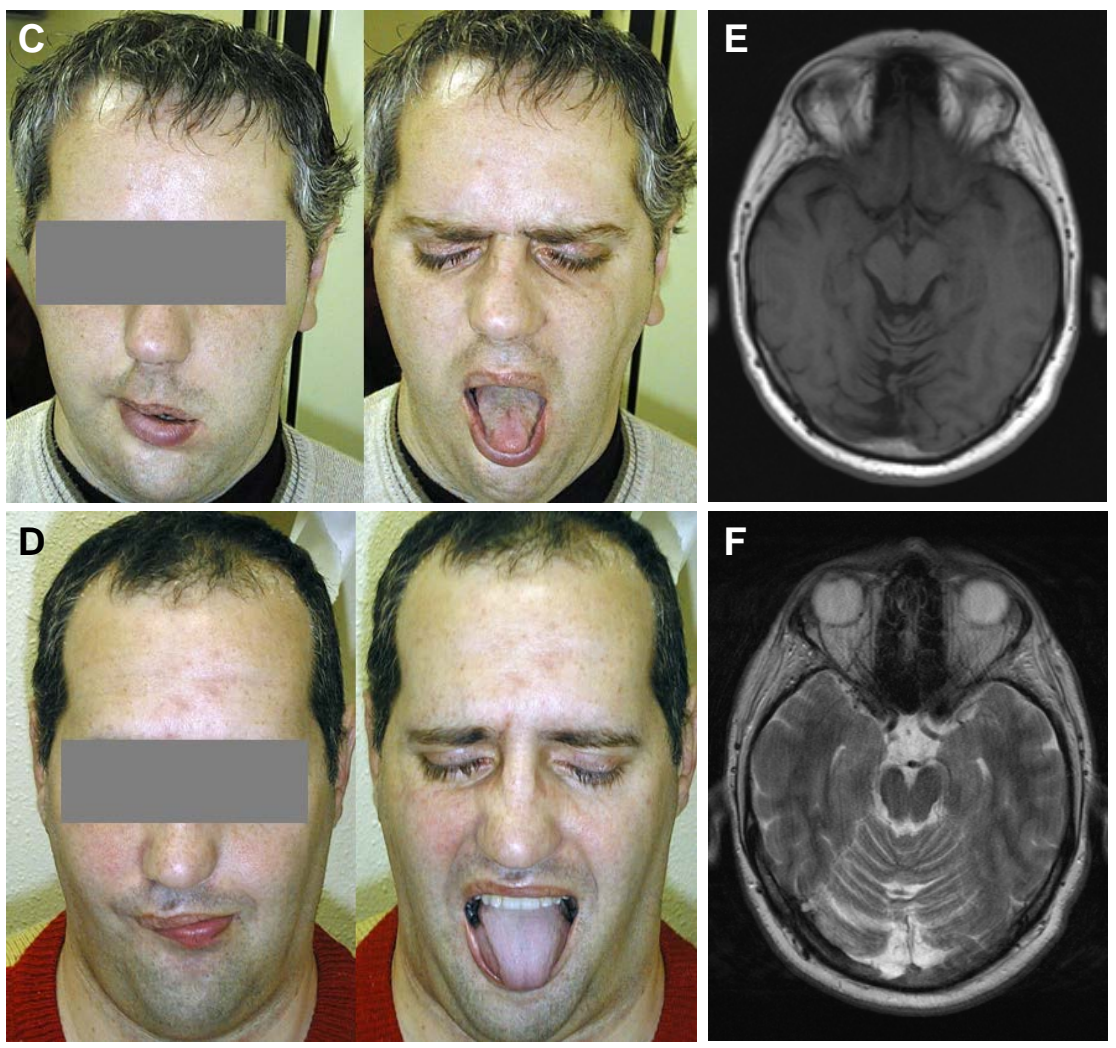
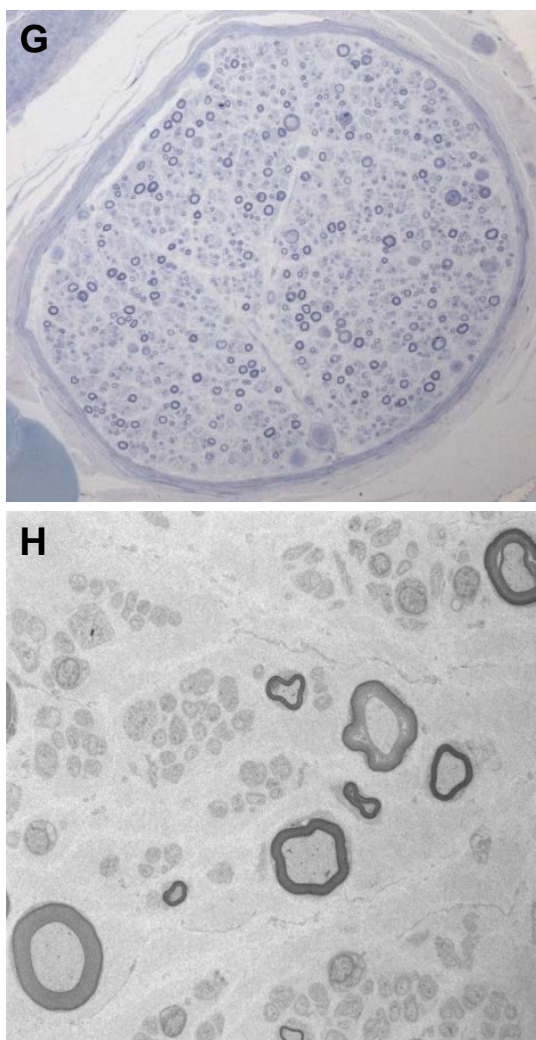
Supplementary material

SBF1 mutations associated with autosomal recessive axonal neuropathy with cranial nerve involvement

Andreea Manole, ${ }^{* 1,2}$ Alejandro Horga,${ }^{* 1}$ Josep Gamez, ${ }^{3}$ Nuria Raguer, ${ }^{4}$ Maria Salvado, ${ }^{3}$ Beatriz San Millán, ${ }^{5}$ Carmen Navarro, ${ }^{5}$ Alan Pittmann, ${ }^{2}$ Mary M. Reilly, ${ }^{1}$ Henry Houlden. ${ }^{1,2}$

${ }^{1}$ MRC Centre for Neuromuscular Diseases, UCL Institute of Neurology, Queen Square, London, UK. 'Department of Molecular Neuroscience, UCL Institute of Neurology, Queen Square, London, United Kingdom. ${ }^{3}$ Neuromuscular Disorders Unit, Department of Neurology, Hospital Universitari Vall d'Hebron and Universitat Autònoma de Barcelona, VHIR, Barcelona, Spain. ${ }^{4}$ Department of Neurophysiology, Hospital Universitari Vall d'Hebron and Universitat Autònoma de Barcelona, VHIR, Barcelona, Spain. ${ }^{5}$ Department of Neuropathology. Complejo Hospitalario Universitario de Vigo, Vigo, Spain.

*These authors contributed equally to the study.

Correspondence: Dr Josep Gamez. Neuromuscular Disorders Unit, Department of Neurology, Hospital Universitari Vall d'Hebron, Passeig Vall d'Hebron, 119-135, 08035 Barcelona, Spain. Email: josepgamez.bcn@gmail.com. 
Supplementary Table 1. Nerve conduction studies of patients II:3 and II:2

\begin{tabular}{llllll}
\hline Patient & II:3 & II:3 & II:2 & II:2 & Normal \\
Age at examination & $20 y$ & $48 y$ & $21 y$ & $49 y$ & values
\end{tabular}

Right / left side

Right Right Left Right Left Right Left

Motor NCS

Facial nerve, 00

DML, ms

D CMAP, $\mathrm{mV}$

$3.9 \quad 4.0$

$\leq 3.5$

$0.4 \quad 0.3$

$\geq 2.0$

Median nerve, APB

DML, ms

2.6

3.5

3.0

$\leq 3.9$

DIP CMAP, mV

7.2

$5.7 / 5.5$

9.2

4.3

$\geq 3.7$

MNCV, $\mathrm{m} / \mathrm{s}$

55.3

61.2

53.1

$8.2 / 7.0$

$\geq 51$

F wave, ms (\%)

26.1 (20)

49

$\leq 31$

Ulnar nerve, ADM

DML, ms

D/P CMAP, mV

2.6

3

NR

MNCV, $\mathrm{m} / \mathrm{s}$

5.2

4.2/3.1

2.7

3.6

$\leq 3.3$

60

49.1

4.5

$6.2 / 5.1$

$\geq 3.3$

F wave, ms (\%)

$28.3(100)$ -

58.5

49.2

$\geq 50$

27.2 (47)

Peroneal nerve, EDB

DML, ms

5.7

NR

NR

6.1

6.6

NR

5.8

$\leq 6.5$

DIP CMAP, mV

NR

NR

2.5

4.9

NR

$0.1 / 0.1$

$\geq 2.5$

MNCV, $\mathrm{m} / \mathrm{s}$

NR

NR

41.9

40.1

NR

34.1

$\geq 42$

Peroneal nerve, TA

DML, ms

8

5.4

4.8

$\leq 5.0$

D CMAP, $\mathrm{mV}$

$<0.1$

$<0.1$

0.1

$\geq 2$

Tibial nerve, TA

DML, ms

9

9.8

NR

8.4

$\leq 6.0$

D/P CMAP, mV

$<0.1 /<0.1$

$<0.1 /<0.1$

NR

$<0.1$

$\geq 1.8$

32.6

30.5

NR

34.5

$\geq 40$

Sensory NCS

Radial nerve

SNAP, $\mu \mathrm{V}$

5

4.5

8.2

$\geq 15$

SNCV, $\mathrm{m} / \mathrm{s}$

56.1

47.6

$\geq 50$

Median nerve, D3

SNAP, $\mu \mathrm{V}$

$7 \quad 3$

10

3.1

$\geq 7$

SNCV, $\mathrm{m} / \mathrm{s}$

48.2

50

53.8

48.3

$\geq 46$

Ulnar nerve, D5

SNAP, $\mu \mathrm{V}$

$4 \quad 1$

4

3.7

$\geq 5$

$54.5 \quad 43.7$

54.1

46

$\geq 46$

Sural nerve

SNAP, $\mu \mathrm{V}$

11

NR

NR

8

NR

NR

$\geq 8$

42.3

NR

NR

40.5

41.4

NR

NR

$\geq 46$

$\mathrm{ADM}=$ abductor digiti minimi; $\mathrm{AH}=$ abductor hallucis; $\mathrm{APB}=$ abductor pollicis brevis; $\mathrm{D} / \mathrm{P} \mathrm{CMAP}=$ distal/proximal compound muscle action potential; $\mathrm{D} 3=$ third finger; $\mathrm{D} 5=$ fifth finger; $\mathrm{DML}=$ distal motor latency; $\mathrm{EDB}=$ extensor digitorum brevis; $\mathrm{F}$ wave $=$ minimal F wave latency; MNCV = motor nerve conduction velocity; NCS = nerve conduction studies; NR = no response; SNAP = sensory nerve action potential; SNCV = sensory nerve conduction velocity; TA = tibialis anterior; - = not assessed. 
Supplementary Table 2. Electromyography results in patients II:3 and II:2

\begin{tabular}{lcc}
\multicolumn{3}{c}{ Spontaneous activity } \\
\hline $\begin{array}{l}\text { Fibrillation } \\
\text { potentials }\end{array}$ & $\begin{array}{c}\text { Positive sharp } \\
\text { waves }\end{array}$ & $\begin{array}{c}\text { Fascicu- } \\
\text { lations }\end{array}$
\end{tabular}

MUAP morphology

Amplitude Polyphasia Duration
MUAP firing pattern

Recruitment

\section{Patient II:3 (48 y)}

$\mathrm{R}$ Orbicularis oris

R Biceps brachii

$1+$

$1+$

0

$\uparrow$

$$
\uparrow \uparrow
$$

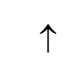

1

R Extensor digitorum com.

$2+$

$2+$

$$
0
$$

0

R First dorsal interosseous

$2+$

$2+$

$$
\uparrow
$$$$
\uparrow \uparrow
$$

$\uparrow$

$\begin{array}{lll}0 & \uparrow & \uparrow\end{array}$

$\mathrm{R}$ Vastus medialis

$$
0
$$$$
1+
$$$$
0
$$

$\mathrm{R}$ Tibialis anterior

R Medial gastrocnemius

$$
0
$$$$
-
$$$$
1+
$$

$$
0
$$$$
0
$$

$$
0
$$

$$
0
$$

$\mathrm{R}$ Extensor digitorum com.

R First dorsal interosseous

0

R Rectus femoris

$$
0
$$$$
0
$$

0

R Tibialis anterior

0

R Medial gastrocnemius

$\begin{array}{lcccc}0 & \uparrow & \uparrow & \uparrow & 1 \\ 0 & \uparrow & \uparrow & \uparrow & 1 \\ 0 & \uparrow & \uparrow & \uparrow & 1 \\ 0 & - & - & - & 0 \\ 0 & - & - & - & 0 \\ 0 & \uparrow & \uparrow & \uparrow & 1 \text { MUAP }\end{array}$

$\mathrm{R}=$ right; com = communis; spontaneous activity = 0 none, 1+ mild, 2+ moderate, 3+ many; MUAP = motor unit action potential; MUAP morphology = N normal, $\uparrow$ increased, - not evaluable; recruitment $=3$ normal, 2 moderately reduced, 1 severely reduced, 0 no recruitment. 
Supplementary Table 3. Whole-exome sequencing results and variant filtering.

\begin{tabular}{|lll}
\hline Individuals & II:3 & II:1 \\
Total no. of reads & $75,483,464$ & $75,671,510$ \\
30x coverage & $64.1 \%$ & $69.1 \%$ \\
20x coverage & $80.5 \%$ & $82.1 \%$ \\
10x coverage & $94.3 \%$ & $93.3 \%$ \\
2x coverage & $99.3 \%$ & $98.6 \%$ \\
Exonic variants & 22,828 & 22,661 \\
Synonymous variants excluded & 11,461 & 11,233 \\
MAF <0.5\% (ExAC, EVS and 1000G) & 613 & 132 \\
Rare variants under AR or XL model & 188 & - \\
Variants in neuropathy-related genes & $3(S B F 1$ gene) & - \\
\hline
\end{tabular}

$\mathrm{AR}=$ autosomal recessive; EVS = Exome Variant Server (http://evs.gs.washington.edu/); ExAC = Exome Aggregation Consortium database (http://exac.broadinstitute.org/); MAF = minor allele frequency; $\mathrm{XL}=\mathrm{X}$-linked; $1000 \mathrm{G}=1000$ Genomes databases (1000G; http://www.1000genomes.org). 


\begin{tabular}{|c|c|c|c|}
\hline \multicolumn{4}{|c|}{ Supplementary Table 4. Sequence variants in SBF1. } \\
\hline Chr 22 (GRCh37/hg19) & g.50903679G >C & g.50900820_50900821del & g.50886828G $>A$ \\
\hline cDNA change & c. $1168 C>G$ & c.2209_2210del & c. $5197 \mathrm{C}>\mathrm{T}$ \\
\hline Protein change & p.Arg390Gly & p.Leu737Glufs*3 & p.Arg1733Cys \\
\hline Exon & 11 & 19 & 38 \\
\hline rs ID & $\mathrm{n} / \mathrm{r}$ & $\mathrm{n} / \mathrm{r}$ & rs199972466 \\
\hline MAF EXAC & $\mathrm{n} / \mathrm{r}$ & $\mathrm{n} / \mathrm{r}$ & $0.05276 \%$ \\
\hline Grantham & 125 & - & 180 \\
\hline PhyloP & 5.47 & $4.48,0.176$ & 4.69 \\
\hline GERP & 4.49 & $4.39,-1.78$ & 3.61 \\
\hline SIFT & Deleterious & $\mathrm{n} / \mathrm{a}$ & Deleterious \\
\hline PolyPhen2 & Probably damaging & $\mathrm{n} / \mathrm{a}$ & Probably damaging \\
\hline Mutation Taster & Disease causing & Disease causing & Disease causing \\
\hline \multicolumn{4}{|c|}{$\begin{array}{l}\text { Esembl reference sequences for SBF1: ENSG00000100241; ENST00000380817. ExAC = Exome Aggregation Consortium } \\
\text { (http://exac.broadinstitute.org/); GERP = Genomic Evolutionary Rate Profiling score (https://genome.ucsc.edu/ ); Grantham = } \\
\text { Grantham Score (supplementary reference 7); MAF = minor allele frequency; Mutation Taster = Mutation Taster v2 } \\
\text { (http://www.mutationtaster.org/); PhyloP = PhyloP basewise conservation score derived from multiple sequence alignment of } 46 \\
\text { vertebrate species (https://genome.ucsc.edu/); PolyPhen2 = Polymorphism Phenotyping v2 (http://genetics.bwh.harvard.edu/ } \\
\text { pph2/); rs ID = reference single nucleotide polymorphism identifier; SIFT = Sorting Intolerant From Tolerant algorithm } \\
\text { (http://sift.jcvi.org/); } \mathrm{n} / \mathrm{r}=\text { not reported; } \mathrm{n} / \mathrm{a}=\text { not available. }\end{array}$} \\
\hline
\end{tabular}




\begin{tabular}{|c|c|c|c|}
\hline Source & Present study & $\begin{array}{l}\text { Mégarbané } 2010 \\
\text { Valente } 2016\end{array}$ & $\begin{array}{l}\text { Bohlega 2011, } \\
\text { Alazami } 2014\end{array}$ \\
\hline Patients & 2 siblings & 2 siblings & 3 siblings \\
\hline Ethnicity & Spanish & Syrian & Saudi Arabian \\
\hline SBF1 mutation/s & $\begin{array}{c}\text { p.Arg390Gly } \\
\text { p.Leu737Glufs*3 }\end{array}$ & $\begin{array}{l}\text { p.Leu335Pro } \\
\text { homozygous }\end{array}$ & $\begin{array}{l}\text { p.Asp443Asn } \\
\text { homozygous }\end{array}$ \\
\hline Age of onset & $4-9 y$ & $2-7 y$ & Infancy \\
\hline First symptoms & Gait difficulties & $\begin{array}{l}\text { Microcephaly (2 y), } \\
\text { strabismus (4 y), gait } \\
\text { difficulties/fatigue (7 y) }\end{array}$ & $\begin{array}{l}\text { Syndactyly, strabismus, } \\
\text { cognitive delay, limb } \\
\text { weakness }(10-20 \text { y) }\end{array}$ \\
\hline Hearing loss & Yes & - & - \\
\hline Nystagmus & Yes & - & - \\
\hline Strabismus & - & Yes & Yes \\
\hline Ophthalmoparesis & Yes (1) & Yes & Yes \\
\hline Absent pupil reactivity & - & Yes & Yes (1) \\
\hline Facial weakness & Yes & Yes & Yes \\
\hline Tongue weakness & Yes & - & - \\
\hline Abnormal gag reflex & Yes & - & Yes (1) \\
\hline Dysarthria & Yes & Yes & Mild (1) \\
\hline Dysphagia & Yes & Yes & Yes (1) \\
\hline Oromandibular dystonia & - & Yes (1) & - \\
\hline $\begin{array}{l}\text { Distal-predominant muscle } \\
\text { wasting and weakness }\end{array}$ & Yes & Yes & Yes \\
\hline Distal-predominant sensory loss & Yes & - & Yes \\
\hline Deep tendon reflexes & Absent & Absent & Absent \\
\hline Foot drop & Yes & Yes (1) & Yes (1) \\
\hline Pes cavus I planus & Mild pes cavus & Pes cavus & - \\
\hline Intellectual impairment & IQs 83, 85 & Yes & IQs 47, 49, 50 \\
\hline Gynecomastia & Yes & - & - \\
\hline Microcephaly & - & Yes & Yes \\
\hline Syndactyly & - & - & Yes \\
\hline Short stature & - & - & Yes (2) \\
\hline Lumbar hyperlordosis & Yes & - & - \\
\hline Elongated face / wide philtrum & - & Yes & - \\
\hline Mild joint laxity / thumb sign & - & Yes & - \\
\hline Respiratory involvement & Yes (1) & - & - \\
\hline
\end{tabular}




\begin{tabular}{|lccc|}
\hline $\begin{array}{l}\text { Nerve conduction studies } \\
\text { and electromyography }\end{array}$ & $\begin{array}{c}\text { Motor and sensory } \\
\text { axonal neuropathy }\end{array}$ & $\begin{array}{c}\text { Motor and sensory } \\
\text { axonal neuropathy }\end{array}$ & $\begin{array}{c}\text { Motor and sensory } \\
\text { axonal neuropathy; } \\
\text { mildly reduced CVs (1) }\end{array}$ \\
$\begin{array}{l}\text { Sural nerve biopsy } \\
\text { Brain MRI }\end{array}$ & $\begin{array}{l}\text { Mild cerebellar atrophy } \\
\text { Axonal neuropathy (1) }\end{array}$ & $\begin{array}{c}\text { Fork and bracket sign; } \\
\text { dysplastic left cerebellar } \\
\text { cortex (1); brain atrophy (1) }\end{array}$ & Brain atrophy (2) \\
\hline $\begin{array}{l}\text { The numbers in brackets indicate the number of individuals showing the clinical feature (when lower than the total number of } \\
\text { affected individuals). }\end{array}$ &
\end{tabular}


Supplementary Figure. Sanger sequencing electropherograms and resulting SBF1 genotypes (Esembl reference sequence ID ENST00000380817).

Reference

cDNA sequence

cDNA position with

sequence variant

I:1, unaffected

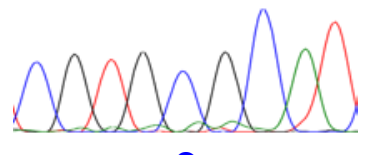

C



$\mathbf{C} / \mathbf{G}$

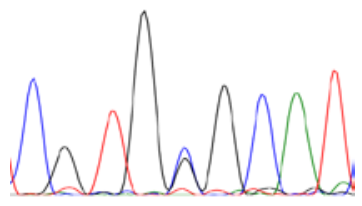

C/G
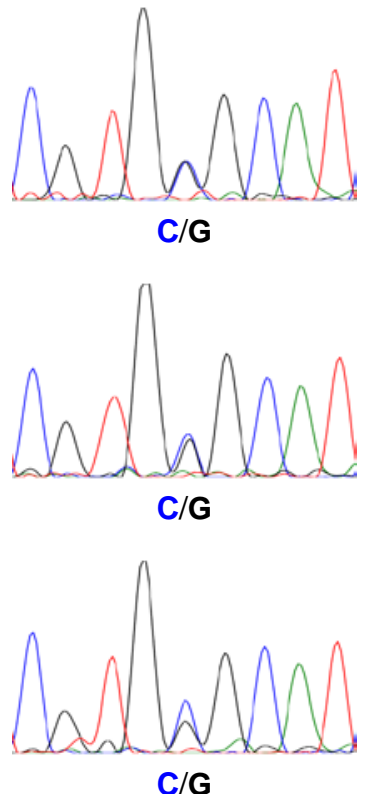

II:4, unaffected
C C A A T C T G A G T C

c.2209_2210

c.5197

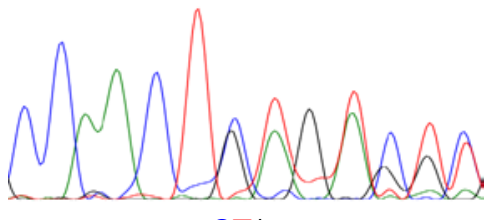

CT/--
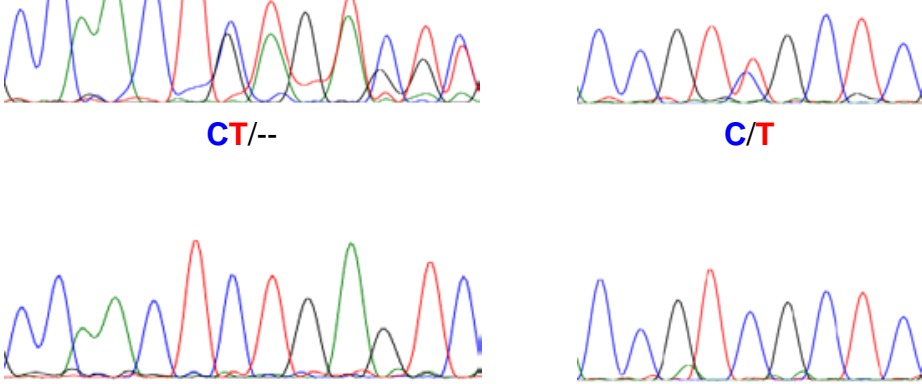

CT

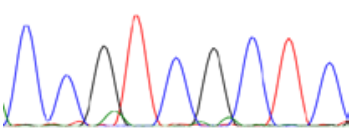

C

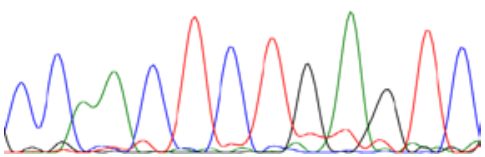

CT

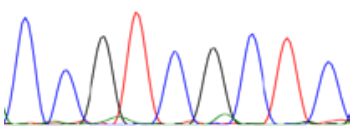

C

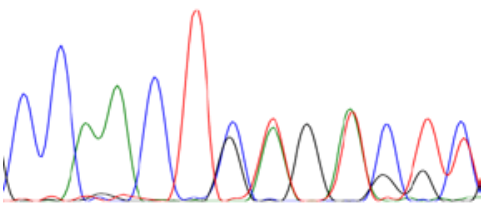

CT/--
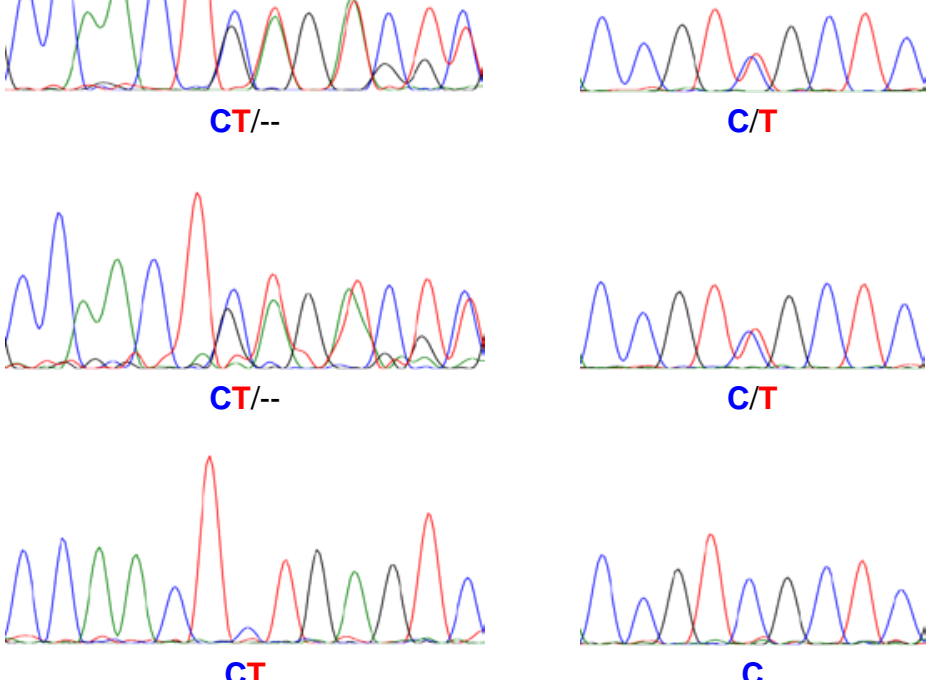


\section{Supplementary methods}

Sanger sequencing. The coding regions and flanking intronic regions of SBF1 (Ensembl reference sequences: ENSG00000100241; ENST00000380817) were PCR-amplified using PCR Master Mix (Roche). Primer sequences and PCR conditions are shown below. PCR products were cleaned up using the ExoSAP-IT treatment (Affymetrix). Sequencing reactions were performed using Big Dye Terminator v3.1 Cycle Sequencing Kit (Applied Biosystems) and products were purified using the Sephadex G50 filtration kit (Thermo Scientific ABgene). DNA fragments were separated on an ABI3730XL automatic DNA sequencer (Applied Biosystems). The resulting sequences were analysed with SeqScape software v2.5 (Applied Biosystems).

\begin{tabular}{|c|c|c|}
\hline \multicolumn{3}{|c|}{ Primer sequences used for PCR and Sanger sequencing of SBF1 gene } \\
\hline Exon & Forward / reverse & DNA Sequence \\
\hline 11 & Forward & T T G G G G T G T G G A G A A G C T C \\
\hline 11 & Reverse & ATGCGCTTATCTCCTACCCC \\
\hline 19 & Forward & C T C A T G C G T G T G G T G C C G \\
\hline 19 & Reverse & GAAGAAACTGTGGGCATGGG \\
\hline 38 & Forward & C CAAGTCCCAACCTCCTGT \\
\hline 38 & Reverse & $A C C A G T T C G A C A C C C C A A A$ \\
\hline
\end{tabular}

\begin{tabular}{|c|c|c|}
\hline \multicolumn{3}{|l|}{ PCR protocol } \\
\hline Temperature $\left({ }^{\circ} \mathrm{C}\right)$ & Time (min) & Number of cycles \\
\hline 94 & 01:00 & \\
\hline 94 & $00: 30$ & \\
\hline 70 & $00: 30$ & 乙 $x 8$ \\
\hline 72 & $00: 30$ & \\
\hline 94 & $00: 30$ & \\
\hline $70^{*}$ & $00: 30$ & 女 $x 16$ \\
\hline 72 & $00: 30$ & \rfloor \\
\hline 94 & $00: 30$ & \\
\hline 65 & $00: 30$ & Y x14 \\
\hline 72 & $00: 30$ & - \\
\hline 72 & 05:00 & \\
\hline
\end{tabular}

* Reducing temperature in each cycle 
Whole-exome sequencing. The exomes of individuals II:3 and II:1 were enriched using the Nextera Rapid Capture Exome kit (Illumina) and sequenced on the HiSeq 2500 platform (Illumina). The resulting 100 base pairs paired-end sequence reads were mapped against the human reference genome assembly 19 (GRCh37) with the Burrows-Wheeler Aligner package ${ }^{1}$ and read duplicates were removed with Picard (http://broadinstitute.github.io/picard/). Variant calling and indel realignments were performed with the Genome Analysis Toolkit (GATK) ${ }^{2}$ and variants were submitted to ANNOVAR for annotation. ${ }^{3}$

Bioinformatic analysis. cDNA and protein sequence variants are described in accordance with the recommendations of the Human Genome Variation Society (http://www.hgvs.org) using Ensembl ENSG00000100241; ENST00000380817 as the reference sequences (http://www.ensembl.org/). Evolutionary conservation of nucleotides was assessed using PhyloP (46 vertebrate species) and GERP scores, ${ }^{4,5}$ which were accessed through the UCSC Genome Browser (https://genome.ucsc.edu/) using genomic coordinates from GRCh37/hg19. Grantham scores were used to assess the physicochemical nature of the amino acid substitutions. ${ }^{6}$ In silico analyses of sequence variants were performed using the following pathogenicity prediction tools: SIFT (http://sift.jcvi.org/), ${ }^{7}$ PolyPhen-2 (http://genetics.bwh.harvard.edu/ pph2/) ${ }^{8}$ and Mutation Taster version 2 (http://www.mutationtaster.org/). ${ }^{9}$

\section{Supplementary references}

1. Li H, Durbin R. Fast and accurate short read alignment with Burrows-Wheeler transform (2009). Bioinformatics 25:1754-1760.

2. McKenna A, Hanna M, Banks E, et al. The Genome Analysis Toolkit: a MapReduce framework for analyzing next-generation DNA sequencing data (2010). Genome Res 20:1297-1303.

3. Wang K, Li M, Hakonarson $\mathrm{H}$. ANNOVAR: functional annotation of genetic variants from highthroughput sequencing data (2010). Nucleic Acids Res 38:e164.

4. Pollard KS, Hubisz MJ, Rosenbloom KR, Siepel A. Detection of nonneutral substitution rates on mammalian phylogenies (2010). Genome Res 20:110-121. 
5. Cooper GM, Stone EA, Asimenos G, Green ED, Batzoglou S, Sidow A. Distribution and intensity of constraint in mammalian genomic sequence (2005). Genome Res 15:901-913.

6. Grantham R. Amino acid difference formula to help explain protein evolution (1974). Science 185:862-864.

7. Ng PC, Henikoff S. SIFT: Predicting amino acid changes that affect protein function (2003). Nucleic Acids Res 31:3812-3814.

8. Adzhubei IA, Schmidt S, Peshkin L, et al. A method and server for predicting damaging missense mutations (2010). Nat Methods 7:248-249.

9. Schwarz JM, Cooper DN, Schuelke M, Seelow D. MutationTaster2: mutation prediction for the deep-sequencing age (2010). Nat Methods 11:361-362. 